\title{
Anencefalia: um estudo epidemiológico de treze anos na cidade de Pelotas
}

\author{
Anencephaly: thirteen years of epidemiological \\ study in Pelotas city
}

Ricardo Ramires Fernández 1

Daniela Zílio Larentis 2

Tiago Fontana 3

Gustavo Pêgas Jaeger 3

Patrick Botelho Moreira 1

Gilberto de Lima Garcias 2,3

Maria da Graça Martino Roth 3

\footnotetext{
1 Faculdade de Ciências Biológicas da Universidade Católica de Pelotas. Laboratório de Genética Universidade Católica de Pelotas.

Rua Félix da Cunha 412, 96010-000, Pelotas RS. ramiresfernandez@gmail.com

2 Faculdade de Medicina da Universidade Federal de Pelotas.

3 Escola de Medicina da Universidade Católica de Pelotas.
}

Abstract The anencephaly is the most common lethal form among the CNS anomalies. Although isolated cases occur, the anencephaly seems to be caused by multiple factors. This malformation result from closure defects of the anterior neural sulk, during embryonic development. The aim of this study was analyze the frequency, etiology, and the risk factors of this congenital malformation. This population-based case-control study range all births occurred in five maternities of Pelotas city from January, 1st 1990 to December, 122002. Key words Anencephaly, Epidemiology, Risks' factors
Resumo A anencefalia é a forma letal mais comum dentre as anomalias do SNC. Apesar dos casos isolados, a anencefalia parece ser de causa multifatorial. Este defeito ocorre devido a um não fechamento da extremidade anterior do sulco neural. Este trabalho, que tem como objetivo estudar a freqüência, a etiologia e os fatores predisponentes desta malformação, é um estudo de caso-controle de base populacional que abrange todos os nascimentos ocorridos nas cinco maternidades da cidade de Pelotas, durante o período de 1o de janeiro de 1990 a 31 de dezembro de 2002. Palavras-chave Anencefalia, Epidemiologia, Fatores de risco 


\section{Introdução}

Os defeitos congênitos são estados patológicos determinados por fatores causais que aparecem concomitantemente ao nascimento, ou seja, antes, durante ou depois da concepção. $\mathrm{O}$ termo congênito significa que o defeito básico está presente, mas não necessariamente reconhecido no nascimento, podendo ser malformações internas ou funcionais que só se manifestam com o avanço da idade (Bianco, 1996).

A freqüência de malformações congênitas em recém-nascidos $(\mathrm{RN})$ varia muito de acordo com o critério e com a metodologia empregados em cada trabalho, sendo considerada aceitável uma freqüência aproximada de 3\%. Nos primeiros 6 meses de funcionamento do Programa de Monitorização de Malformações congênitas no Hospital de Clínicas de Porto Alegre, no período de fevereiro a julho de 1983, quando nasceram 1.197 crianças, 42 recém-nascidos apresentaram, pelo menos, uma malformação clinicamente significante (3,5\%) (Giugliani, 1985).

A ocorrência de malformações congênitas pode estar relacionada a agentes teratogênicos. Para Borges-Osório \& Robinson (2001), agentes teratogênicos são aqueles que agem sobre o organismo em formação, produzindo anomalias características ou gerais, ou aumentando a incidência de uma anomalia na população. Os principais agentes teratogênicos ou teratógenos são as radiações, os vírus, as drogas e as doenças maternas. $\mathrm{O}$ efeito teratogênico desses agentes depende de vários fatores, tais como: tempo de exposição ao teratógeno, dosagem do teratógeno, genótipo materno, genótipo e suscetibilidade do embrião, atividade enzimática do feto, interação entre teratógenos e especificidade dos mesmos. No entanto, para Giugliani (1985), as malformações congênitas são etiologicamente heterogêneas. Recentemente, estimou-se que a maior parte das malformações são de etiologia desconhecida ou multifatorial, enquanto que em apenas $20 \%$ dos casos pode-se identificar o fator causal genético, materno ou ambiental.

Dentre as anomalias que afetam o sistema nervoso central, a Anencefalia - ausência parcial do encéfalo - é o defeito mais grave e, também, o mais comum (Moore \& Persaud, 1998). Esse defeito resulta quando ocorre uma falha de fechamento do tubo neural durante a $3 \underline{a}$ e $4 \underline{a}$ semana de desenvolvimento levando a uma perda fetal, nativivos ou morte neonatal. Nos
EUA, por exemplo, a prevalência de anencefalia varia de 1 para cada 1.000 ou 2.000 nascimentos, com aumento da freqüência do Oeste para o Leste (Best, 2002). Em outro estudo realizado na cidade de Bauru (SP), de junho de 1990 a dezembro de 1992, verificou-se uma prevalência de 0,6 malformado para cada 1.000 nascimentos (Bianco, 1996). Quanto à raça, os caucasóides são mais afetados que os negróides, bem como as mulheres são mais afetadas que os homens (2:1). A sazonalidade também é relevante no nascimento de anencéfalos, sendo mais freqüente quando as concepções se dão no outono. Em relação à idade materna existe um maior acometimento em filhos de mulheres com mais de 40 anos e com nível socioeconômico desfavorável. Um aspecto relevante na prevenção de anencefalia é o suplemento de ácido fólico na dieta materna no período da concepção, reduzindo em 50\% estas malformações. Assim, a anencefalia como outras formas de defeitos do tubo neural geralmente seguem um modelo multifatorial de transmissão com a interação de múltiplos genes bem como fatores ambientais. Esta anomalia pode ser diagnosticada no período pré-natal através da dosagem de alfa-fetoproteína no soro materno ou no líquido amniótico ou, ainda, por ultra-som (Best, 2002).

\section{Material e métodos}

O presente projeto teve início em 1990 e, desde então, vem sendo realizado nos berçários de todos os hospitais de Pelotas (Beneficência Portuguesa, Hospital São Francisco de Paula, Hospital Escola da FAU, Santa Casa de Misericórdia de Pelotas e Hospital Miguel Piltcher), segundo metodologia descrita no Manual Operacional ECLAMC-MONITOR, edição de 1982.

Consideraram-se no programa todos os nascimentos, vivos ou mortos, de $500 \mathrm{~g}$ ou mais de peso, ocorridos no hospital a partir de seu ingresso no programa. Não foram considerados os nascimentos ocorridos fora do hospital e que posteriormente ingressaram no setor de neonatologia. Todos os recém-nascidos, vivos ou mortos, foram examinados clinicamente, em busca de malformações, e esta observação se estendeu até sua alta hospitalar.

Neste estudo, todos os recém-nascidos foram examinados por pediatras, segundo protocolo de cada maternidade. Um segundo exame foi feito por acadêmicos do curso de medicina, 
especialmente treinados para a detecção de anomalias congênitas. Os recém-nascidos portadores de malformação foram re-examinados por especialistas do projeto.

Na definição e descrição das malformações, considerou-se malformação toda alteração morfológica, interna ou externa, clinicamente diagnosticável, com um aceitável grau de certeza antes da alta hospitalar, em todo o recémnascido.

Para os recém-nascidos com malformações (caso) tomou-se um neonato vivo (controle) pareado a ele. Foi controle o recém-nascido vivo não malformado e de igual sexo que nasceu no hospital imediatamente depois do malformado. Neste estudo, para cada recém-nascido com anencefalia (caso) foram tomados quatro controles.

Para a formação do banco de dados foram tomados todos os dados obtidos, através do preenchimento dos formulários modelo ECLAMC, codificados e, posteriormente, tabulados pelo programa SPSS for Windows versão 10.0. Para análise dos dados foram utilizados o teste $t$ de Student, o $\mathrm{X}^{2}$ e Odds Ratio, sendo consideradas significativas as diferenças com $\mathrm{P}<0,05$.

\section{Resultados e discussão}

Dos 71.500 nascimentos ocorridos nos cinco hospitais-maternidade, 980 recém-nascidos eram portadores de algum tipo de anomalia congênita (1,37\%). Destes, 49 (5\%) tiveram diagnóstico de anencefalia, determinando uma prevalência de 0,68/1.000 nascimentos, diferindo dos resultados achados por Canfield et al. (1996), World Atlas of Birth Defects (2003), Botto et al. (1999), Hendrick et al. (1999), os quais acharam uma prevalência de 0,38, 0,86, 1,00 e 1,97 respectivamente e concordando com os dados de Limb \& Holmes (1994), que durante os anos de 1988 a 1990 encontraram uma prevalência de 0,63 para cada 1.000 nascimentos e Merlos (1995) que encontrou 0,66\%.

Estudos realizados por Nazer et al. (2001), compreendendo trinta anos de vigilância epidemiológica, mostram uma taxa de anencefalia no ECLAMC-Chile durante o período de 19691999 de 7,16 por 10.000, significativamente maior da observada no ECLAMC de 6,06. Em publicação anterior, Castilla \& López-Camelo (1990) relatam que no começo do monitoramento feito pelo ECLAMC, Chile, compreendido no período de 1967-1973, a freqüência de anencéfalos era menor. Porém, a partir do ano de 1974 até 1999 esta freqüência supera significativamente a do resto do ECLAMC. Segundo os autores este fenômeno é difícil de interpretar já que tal estudo colaborativo começou a registrar malformações em natimortos a partir de 1978 e, como mais da metade dos portadores de anencefalia nascem mortos, as diferenças anteriores a 1978 podem se dever à variação de grau de registro e a diferenças em proporção de casos de DTN nascidos mortos. É sabido que os sistemas de registros se auto-educam e, conseqüentemente, melhoram sua cobertura, refletindo em tendências seculares em aumento.

Resultados importantes, como o sexo dos $\mathrm{RN}$, foram observados, sendo acometido o dobro de indivíduos do sexo feminino (2:1) com $65,3 \%$, concordando com Canfield et al. (1996), Verma et al. (1991) e Loncarek et al. (2001), cujos estudos também encontraram aproximadamente o dobro de recém-nascidos com anencefalia do sexo feminino. Foram significativos $(\mathrm{P}<0,05)$ (Tabela 1) os resultados correspondentes a ocorrência de parto gemelar, ocorrendo em $8,2 \%$ dos casos e 1,5\% dos controles, cujos valores são aproximados aos encontrados no The Victorian Congenital Malformation/Birth Defects Register (http://www.dhs.vic.gov.au/phd/hce/peri/conmal/sec1.htm). Dos resultados estatísticos encontrados para a sazonalidade, as maiores taxas foram encontradas no outono e primavera ambos com 34,7\% dos casos. Tanto os trabalhos de Abrate et al. (1990) e Ramos et al. (1981) encontraram menor número de nascimentos de anencéfalos durante o verão.

A prevalência de natimortos $(\mathrm{P}<0,001)$ foi observada nos casos com percentual de $49 \%$ e $0 \%$ nos controles, conforme também achado por Shaw et al. (1994), cujo estudo demonstrou 45\% de natimortos entre 193 casos de anencefalia, durante 5 anos de monitorização na Colômbia e contrastando com Gucciard et al. (2002), os quais acharam um índice de 4,4\%. Receberam alta em óbito $(\mathrm{P}<0,001) 81,6 \%$ dos casos e $0,5 \%$ dos controles. Dos nascidos com anencefalia, apresentaram peso normal, baixo e muito baixo $18,4 \%, 26,5 \%$ e $32,7 \%$ nos casos e, $88,6 \% ; 8,5 \%$ e $0,5 \%$ dos controles, respectivamente $(\mathrm{P}<0,001)$ (Tabela 1$)$, discordando com o estudo em Victoria que achou respectivamente $12,2 \%, 47,1 \%$ e $33,5 \%$, predominando, dessa forma, recém-nascidos com baixo peso. Quanto ao parto $(\mathrm{P}<0,001)$ (Tabela 1$)$, a cesariana foi eletiva em $55,1 \%$ dos casos ante a $26,4 \%$ dos controles. 


\begin{tabular}{|c|c|c|c|c|}
\hline Variável & $\operatorname{Casos}^{\star}(n=49)$ & Controles $^{\star}(n=201)$ & ORB $(\mathrm{IC}=95 \%)$ & $\mathbf{P}$ \\
\hline \multicolumn{5}{|l|}{ Gemelaridade } \\
\hline Com gemelar & $45(91,8 \%)$ & $195(97 \%)$ & 1 & $<0,05$ \\
\hline Sem gemelar & $4(8,2 \%)$ & $3(1,5 \%)$ & $0,17(0,03-0,96)$ & \\
\hline \multicolumn{5}{|l|}{ Peso } \\
\hline Muito baixo peso & $16(32,7 \%)$ & $1(0,5 \%)$ & 1 & \\
\hline Baixo peso & $13(26,5 \%)$ & $17(8,5 \%)$ & $20,92(2,31-479,26)$ & $<0,001$ \\
\hline Normal & $9(18,4 \%)$ & $178(88,6 \%)$ & $316,44(36,17-7154,92)$ & \\
\hline \multicolumn{5}{|c|}{ Número de natimortos nas gestas } \\
\hline Sem natimortos & $21(42,9 \%)$ & $181(90 \%)$ & 1 & $<0,001$ \\
\hline Com natimortos & $19(38,8 \%)$ & $8(4 \%)$ & $0,05(0,02-0,14)$ & \\
\hline \multicolumn{5}{|c|}{ Número de abortos espontâneos } \\
\hline Sem & $31(63,3 \%)$ & $161(80,1 \%)$ & 1 & $=0,06$ \\
\hline Com um ou mais & $7(14,3 \%)$ & $15(7,5 \%)$ & $0,41(0,14-1,23)$ & \\
\hline \multicolumn{5}{|l|}{ Idade paterna } \\
\hline $10-19$ anos & $3(6,1 \%)$ & $11(5,5 \%)$ & 1 & \\
\hline 20-40 anos & $30(61,2 \%)$ & $163(81,1 \%)$ & $1,48(0,31-6,24)$ & $<0,05$ \\
\hline 41-54 anos & $9(18,4 \%)$ & $15(7,5 \%)$ & $0,45(0,07-2,53)$ & \\
\hline \multicolumn{5}{|l|}{ Malformados na família } \\
\hline Com malformados & $16(32,7 \%)$ & $25(12,4 \%)$ & 1 & $<0,001$ \\
\hline Sem malformados & $30(61,2 \%)$ & $166(82,6 \%)$ & $0,28(0,13-0,63)$ & \\
\hline \multicolumn{5}{|l|}{ Consangüinidade familiar } \\
\hline Com consangüinidade & $6(12,2 \%)$ & $9(4,5 \%)$ & 1 & $<0,05$ \\
\hline Sem consangüinidade & $36(73,5 \%)$ & $180(89,6 \%)$ & $0,3(0,09-1,02)$ & \\
\hline \multicolumn{5}{|l|}{ Antepassados } \\
\hline Caucasóides & $36(73,4 \%)$ & $118(58,7 \%)$ & 1 & \\
\hline Negróides & $1(2 \%)$ & $21(10,4 \%)$ & $6,41(0,86-132,22)$ & $<0,05$ \\
\hline \multicolumn{5}{|l|}{ Zona } \\
\hline Rural & $10(20,4 \%)$ & $15(7,5 \%)$ & 1 & $<0,05$ \\
\hline Urbana & $39(79,6 \%)$ & $182(90,5 \%)$ & $3,11(1,19-8,04)$ & \\
\hline
\end{tabular}

* Diferenças em totais devido a perda de informações.

O número de abortos espontâneos em gestas prévias (Tabela 1) ocorreu em $14,3 \%$ das mães dos casos e em 7,5\% das mães dos controles, conforme a teoria de Knox (1978) os restos trofoblásticos de um aborto prévio interferem na próxima gestação no que se refere a anomalias do tubo neural. A idade materna com maior prevalência tanto em casos como em controles foi entre a faixa de 20 a 34 anos com $53,1 \%$ e $68,7 \%$, respectivamente; de acordo com o estudo realizado em Victoria (2002), que também não achou associação significativa entre a idade materna, encontrada por este estudo, e a ocorrência de anencefalia. Com referência ao número de natimortos na gesta atual e/ou anterior(es) $(\mathrm{P}<0,001)$ (Tabela 1), 42,9\% das mães dos casos não apresentaram natimortos e $38,8 \%$ apresentaram um ou mais, sendo que, dos controles, $90 \%$ e $4 \%$, corresponden- tes, diferindo de Ramos et al. (1981), o qual não encontrou resultados estatísticos significativos em seu estudo com 141 malformados.

Quanto à idade paterna $(\mathrm{P}<0,05)($ Tabela 1), $18,4 \%$ dos pais dos casos tinham entre 41 a 54 anos e dos controles, 7,5\%, diferindo de Hall et al. (1988) e Seller (1986), cujos resultados não revelaram alguma diferença estatística significativa para tal dado.

A história familiar de anencefalia $(\mathrm{P}<0,05)$ foi observada em $50 \%$ dos casos e $0 \%$ dos controles, já Merlos (1995) relata um risco de recorrência de $10 \%$ em cada gestação subseqüente, de uma criança com anencefalia. Apresentaram consangüinidade $12,2 \%$ e $4,5 \%$ dos casos e controles, respectivamente, diferindo de Abrate et al. (1990), o qual achou um índice de $4,4 \%$. Quanto à localidade de origem dos pais dos RNs $(\mathrm{P}<0,05)$ (Tabela 1), 79,6\% de casos 
de anencefalia ocorreram na região urbana, enquanto que nos controles houve $90,5 \%$ na mesma região; o estudo de Tambosi (1997) revelou relação direta do aumento do índice do número dos casos de anencefalia com o uso de agrotóxicos na zona rural, registrando um índice de 3 malformados com anencefalia para cada 1.000 nascimentos.

$\mathrm{Na}$ etnia dos antepassados $2 \%$ dos casos apresentavam antepassados negróides ante a 10,4\% dos controles, divergindo de Limb \& Holmes (1994), em que os antepassados negróides eram em torno de 16,2\%. Em relação à ocorrência de malformações na família $(\mathrm{P}<0,001)$ (Tabela 1), havendo em 32,7\% nos casos e 12,4\% nos controles, concordando com Ramos et al. (1981), o qual também encontrou significância entre qualquer malformação congênita na família e a ocorrência de anencefalia.

\section{Colaboradores}

RR Fernández delineou os aspectos a serem abordados na discussão do artigo e sua estruturação. T Fontana participou da elaboração do banco de dados bem como na discussão e estruturação do artigo; DZ Larentis, da estruturação do artigo e discussão; GP Jaeger, da revisão bibliográfica e estruturação do artigo e PB Moreira, da elaboração da discussão do artigo. GL Garcias realizou a revisão crítica do artigo e MGM Roth, a revisão crítica do artigo e supervisão.

\section{Referências bibliográficas}

Abrate M et al. 1990. Epidemiological study of anencephaly in Italy and anatomo-pathological findings in cases observed by us. Pathologica 82(1078):133154.

Best RG 2002. Anencephaly. Medicine Journal 3(2).

Bianco MHBC 1996. Monitorização de malformações congênitas. Ed. Cadernos de Divulgação Cultural, São Paulo.

Borges-Osório MR \& Robinson WM 2001. Genética humana. Artmed, Porto Alegre.

Botto LD, Moore CA, Khoury MJ \& Erickson JD 1999. Neural-tube defects. The New England Journal of Medicine 341:1509-1519.

Canfield MA, Annexes JF, Brender JD, Cooper SP \& Greenberg F 1996. Hispanic origin and neural tube defects in Houston Harris Country Texas. American Journal of Epidemiology 143(1):1-11.

\section{Conclusão}

Assim, devido ao fato de a anencefalia ser uma anomalia de alta natimortalidade mostra-se muito importante um melhor conhecimento acerca de seus fatores de risco bem como de sua correlação com o aparecimento desta. Do ponto de vista epidemiológico, os dados encontrados no presente estudo, em sua maioria, concordam com a literatura mundial, reforçando teorias previamente publicadas. Quanto aos dados que discordam com a revisão bibliográfica, sua importância é relevante por levantar novas questões e hipóteses, as quais poderão servir como base para futuros estudos.
Castilla EE \& López-Camelo JS 1990. The surveillance of birth defects in South America: The search for time clusters. Epidemics. Advances in Mutagenesis Research 2:191-210.

Giugliani R 1985. Medicamentos na gestação e malformações congênitas. Revista do HCPA 5(1):109-112.

Gucciardi E, Pietrusiak M, Reynolds DL \& Rouleau J 2002. Incidence of neural tube defects in Ontario, 1986-1999. Canadian Medical Association 167(3).

Hall JG et al. 1988. Clinical, genetic and epidemiological factors in neural tube defects. American Journal of Human Genetics 43:827.

Hendricks KA, Simpson JS \& Larsen RD 1999. Neural tube defects along the Texas-Mexico border, 19931995. American Journal of Epidemiology 149:11191127.

Knox EG 1978 Fetus-fetus interaction - a model of etiol- 
ogy for anencephaly or spina bifida. Brazilian Medical Journal 1:72.

Limb CJ \& Holmes LB 1994. Anencephaly: changes in prenatal detection and birth status, 1972 through 1990. American Journal of Obstetrics and Gynecology 170(5):1333-1338.

Loncarek K, Mustac E, Frkovic A \& Prodan M 2001. Prevalence of anencephaly in the region of Rijeka, Croatia. European Journal of Epidemiology 17(3):241244.

Merlos MA 1995. Anencefalia. Clínica Pediátrica 2(19):5-27.

Moore KL \& Persaud TVN 1998. Embriologia clínica. Ed. Guanabara Koogan, Rio de Janeiro.

Nazer H, López-Camelo \& Castilla EE 2001. ECLAMC: Estudio de 30 años de vigilancia epidemiológica de defectos de tubo neural en Chile y en Latinoamérica. Rev. Méd. Chile 129(5):531-539.

Ramos JLA et al. 1981. Malformações congênitas: estudo prospectivo de dois anos em três maternidades de São Paulo. Pediatrics 3:20-28.

Seller MJ 1986. Neural tube defects and sex ratios. Lancet 2:227.
Shaw GM, Jensvold NG, Wasserman CR \& Lammer EJ 1994. Epidemiological characteristics of phenotypically distinct neural tube defects among 0.7 milion California births, 1983-1987. Teratology 49(2):143-149.

Tambosi EF 1997. Estratégias de desenvolvimento concernentes com a relação sociedade e natureza: um estudo de caso. Dissertação de mestrado. Programa de PósGraduação em Engenharia de Produção. Universidade Federal de Santa Catarina, Florianópolis.

The Victorian Congenital Malformations/Birth Defects Register. Congenital malformations in Victoria 19831994. Disponível em <http://www.dhs.vic.gov.au/ $\mathrm{phd} /$ hce/peri/conmal/secl.htm $>$. Arquivo capturado em 22 de novembro de 2002.

Verma M, Chatwal J \& Sing D 1991. Congenital malformations - a retrospective study of 10,000 cases. Indian Journal of Pediatrics 58(2):245-252.

WHO - World Health Organization. World Atlas of Birth Defects. Disponível em <http://www.who.int/genomics/about/en/anencephaly.pdf $>$. Arquivo capturado em 20 de abril de 2004.

Artigo apresentado em 19/12/2003

Aprovado em 13/2/2004

Versão final apresentada em 20/2/2004 